
\title{
R Reserach S Suare \\ Detachment Stress Mediated Bioenergetic Switch of Malignant Melanoma Cells Into Anti-Warburg Phenotype
}

\section{WuChing Uen}

Shin Kong Wu Ho-Su Memorial Hospital

\section{TingTing Tseng}

Fu Jen Catholic University

\section{Ching-Po Wu}

Fu Jen Catholic University

ShaoChen Lee (D073798@mail.fju.edu.tw)

Fu Jen Catholic University

\section{Research Article}

Keywords: stress, melanoma cells, phenotype, cancer cells

Posted Date: September 3rd, 2021

DOI: https://doi.org/10.21203/rs.3.rs-855821/v1

License: (c) (i) This work is licensed under a Creative Commons Attribution 4.0 International License. Read Full License 


\section{Abstract}

One of the biological features of cancer cells was their aerobic glycolysis by extensive glucose fermentation to harvest energy, so called Warburg effect. Melanoma is one of the most aggressive human cancers with poor prognosis and high mortality for its high metastatic ability. During the metastatic process, the metastatic tumor cells should survive under detachment stress. However, whether the detachment stress could affect the tumor phenotype was worthy to investigate.

We had established human melanoma A375 cells under detachment stress, which mimicked circulating melanoma. It was shown the detachment stress altered melanoma cell activities, malignancy, and drug sensitivity. In this study, we found that adherent melanoma cells were more sensitive to glucose depletion. However, detachment stress reduced lactate secretion owing to the reduced MCT4 and GLUT1 expressions, the altered glycolytic and respiratory capacities, and the increased superoxide production. Detachment stress also increase the sensitivity of melanoma cells toward blockade of electron transport chains. Investigation of the change in glucose metabolism of melanoma cells under detachment stress would be critical to provide novel molecular mechanism to develop potential therapeutics

\section{Introduction}

Detachment of tumor cells from extracellular matrix (ECM) and survival under anchorage-independent condition was recognized as initial step of tumor metastasis ${ }^{1}$. ECM detachment of normal cells causes cell apoptosis through anoikis signaling ${ }^{2,3}$. However, tumor cells would survive under detachment stress by harvesting the ability of anoikis resistance and anchorage-independent growth ${ }^{4}$. It was suggested that anchorage-independent growth promoted oncogenic transformation through hyperactive signaling pathways associated with epithelial-mesenchymal transition. Many studies had focused on the change of protein expression for malignant metastatic cells under anchorage-independency. However, the understanding how these changes contributed to alternative phenotypes and altered their microenvironment specificity of tumor cells was limited.

Under aerobic condition, normal cells generate ATP from glucose metabolism through coupled mechanisms of glycolysis, tricarboxylic acid cycle, and oxidative phosphorylation. For glycolysis, glucose oxidation by serial enzymes produced pyruvate, which enters the tricarboxylic acid cycle in the mitochondria. These processes further produced the reduced electron carriers $\mathrm{NADH}$ and $\mathrm{FADH}_{2}$, which could be further used in oxidative phosphorylation. On the other hand, tumor cells generate ATP and biosynthetic backbone through the altered metabolism relying mostly on aerobic glycolysis in the presence of abundant oxygen support ${ }^{5-9}$. Otto Warburg suggested the uncoupling of glycolysis with tricarboxylic acid cycle and oxidative phosphorylation led to increased lactate production, so called the Warburg effect. However, if there is any mechanism could actively reverse the feature of Warburg effect in cancer cells, that will be interesting and worthy to be investigated. 
Previously, we had studied the roles of detachment stress at melanoma cells to affect their cell activities, tumor malignancy, heterogeneity, and drug sensitivity ${ }^{10-13}$. In this paper, we also investigate whether the glucose metabolism and mitochondrial respiration were different between adherent and suspended melanoma cells. The drug sensitivities toward different compounds were also changed by detachment stress.

\section{Results}

\section{Detachment stress altered glucose dependency and lactate secretion through downregulation of MCT4 and GLUT1}

Previously, we had shown the reduced cell growth was observed in suspended melanoma cells comparing to that of adherent melanoma cells ${ }^{11}$. Reduced cell growth correlated with the decrease in cell survival or delay in cell proliferation. Thus, we analyzed their DNA contents by propidium iodide staining under different serum concentration (Fig. 1A). Obviously, the S/G2 contents were lower in suspended melanoma cells than that in adherent cells. In addition, decrease in serum concentrations showed reduced S-phase content in both adherent and suspended melanoma cells as seen the contents between the $\mathrm{G} 1$ and $\mathrm{G} 2$ peaks. This is correlated with our previous observation that cell growth was reduced upon cell detachment ${ }^{10}$. And it was reasonable that serum factors would promote cell proliferation by increasing DNA synthesis upon increased serum levels.

In general, cancer cells were more dependent on aerobic glycolysis to obtain energy, so that they would be sensitive to the change in glucose concentration. To examine whether the detachment stress would alter the phenotypes of glucose-dependency, melanoma cells were cultured under different glucose concentration. As seen in Fig. 1B, decreased glucose concentration suppressed the cell proliferation of adherent melanoma cells. Reducing the glucose concentration to $2.5 \mathrm{mM}$ almost blocked the cell growth of adherent cells. However, the cell growth remained similar for suspended melanoma cells under lower glucose concentrations. This indicated that suspended melanoma cells were less sensitive to the decreased glucose supplement. Either reduced glucose demand was presented in suspended melanoma cells or higher efficiency of glucose uptake might counteract the reduced glucose supply. Increased glucose demand owing to increased glycolysis rate could be detected by increased lactate release. Since adherent melanoma cells were more dependent on glucose level, we examined whether the level of lactate secretion was higher in adherent melanoma cells. As seen in Fig. 1C, more lactate secretion from adherent melanoma cells was observed as examining the extracellular lactate content in the conditioned medium under pH7.0. Under alkaline environment (pH8.5), the lactate release achieved at a saturated level ${ }^{14}$ for both adherent and suspended melanoma cells.

Since glucose dependency and lactate secretion level were different in adherent and suspended melanoma cells, we analyzed the gene expressions of carboxylate transporters. As shown in Fig. 2A, qPCR analysis at adherent or suspended melanoma cells showed that MCT4 and GLUT1 expression were 
reduced upon detachment stress. MCT4 was a low-affinity transporter to export lactate from glycolytic cancer cells, and played role as lactate donor in metabolic symbiosis ${ }^{15}$. The reduced MCT4 expression in suspended melanoma cells would result in the reduced lactate secretion than that by adherent melanoma cells (Fig. 1C). GLUT1 was the glucose transporter that was responsible for glucose supply in glycolysis. The reduced GLUT1 expression might corelate with the lower glucose dependency and reduced lactate secretion in suspended melanoma cells. Further analysis at protein expression also supported our explanations. Western blot analysis showed the MCT4 protein level, but not MCT1or MCT2 protein levels, reduced in suspended melanoma cells (Fig. 2B). GLUT1 protein level was also reduced in suspended melanoma cells. These suggested that less lactate secretion would derived from reduced glucose uptake and reduced lactate export.

\section{Detachment stress caused metabolic switch from glycolytic pathway into aerobic pathway}

Since lactate export was reduced, its effect at extracellular acidification would be suppressed. We analyzed the cellular bioenergetics of adherent and suspended melanoma cells using Seahorse Bioscience XF24 extracellular flux analyzer.

Extracellular acidification rate (ECAR) was the indicator of glycolysis. For evaluation of ECAR and glycolysis capacity, either adherent or suspended melanoma cells were initially incubated under glucosefree medium. Extracellular acidification by lactate release was reported as $\mathrm{pH}$ change by the change in emission fluorescence of $\mathrm{pH}$-sensitive fluorophore. After sequential addition of glucose, oligomycin, and 2-deoxyglucose (2DG), the quantity of basal glycolysis, maximal glycolysis, and non-glycolytic acidification were determined. Oligomycin inhibits ATP synthase that reflected the amount of ATP production. 2DG is the glucose analogue that blocks glucose uptake. As seen in Fig. 3A, suspended melanoma cells had lower level of glycolysis, which was consistent with lower lactate secretion (Fig. 1B). Upon addition of oligomycin, blockade of ATP synthease reflected maximal glycolysis, i.e. glycolytic capacity. This suggested that adherent melanoma cells were more glucose-dependent by higher glycolytic capacity than suspended melanoma cells (Fig. 1A).

Oxygen consumption rate (OCR) was the indicator of mitochondrial respiration. For evaluation of $\mathrm{OCR}$, an $\mathrm{O}_{2}$-quenchable fluorophore was used to reflect $\mathrm{O}_{2}$ consumption. Sequentially additions of oligomycin, FCCP, and rotenone were done to evaluate the change in OCR. Oligomycin inhibits ATP synthase that reflected the amount of ATP production. FCCP disrupted mitochondrial $\mathrm{H}^{+}$-gradient that showed maximal respiration. Combined action of rotenone and antimycin $A$ inhibited electron transport chain that revealed the respiration level from non-mitochondrial sources. As seen in Fig. 3B, the basal respiration rate of suspended melanoma cells was higher than that of adherent melanoma cells. The level of nonmitochondrial oxygen consumption was similar for both adherent and suspended melanoma cells (see the level after addition of rotenone and antimycin A). These indicated that the overall ATP production from mitochondria source was higher in suspended melanoma cells. The maximal respiratory capacity 
for suspended melanoma cells was significantly larger than that for adherent cells (Fig. 3B). This suggested that suspended melanoma cells harvested energy extensively from mitochondrial respiration.

Overly, the basal rate and maximal capacity of ECAR were lower but those of OCR were higher for suspended melanoma (Fig. 3A and 3C). The difference between the basal rate and the maximum glycolytic capacity defined as the glycolytic reserve. The glycolytic reserve for adherent and suspended melanoma cells were the same as $80 \%$ of full capacity. We explained that the reduction in glycolytic capacity, but not the enzymatic conversion in glycolytic reactions under detachment stress. Reduced glycose uptake through reduced GLUT1 expression would account for this result.

Increase maximal respiratory capacity was found in suspended melanoma cells (Fig. 3B and 3D). Approximate 4-folded increase in maximal respiratory capacity for melanoma cells under detachment stress. For adherent melanoma cells, the basal and maximal respiratory rates were equal, which indicated that saturated respiratory level and no spare respiratory capacity in adherent melanoma cells. However, basal respiratory rate in suspended melanoma cells showed $46.7 \%$ saturated level (Fig. 3D), which suggested enough spare capacity for energy production through mitochondrial respiration. It was reported that the increase in spare capacity implied the increased capacity of energy production in response to stress ${ }^{16}$. Mitochondrial spare respiratory capacity was the extra capacity to produce energy in response to increased stress and was associated with cellular survival. The preference of metabolic pathways could be estimated by the ratio of OCR to ECAR ${ }^{17}$. As seen in Fig. 3E, the OCR/ECAR ratio in suspended melanoma cells was 3.3-folded higher than that in adherent melanoma cells. This implied the detachment stress enabled metabolic switch of melanoma cells from Warburg phenotype into antiWarburg phenotype.

\section{Detachment stress increased the superoxide production}

Since suspended melanoma cells produced energy mostly through mitochondrial respiration, we examined whether the level of reactive oxygen species (ROS) in suspended melanoma cells was different from that in adherent melanoma cells. ROS include the superoxide anion $\left(\mathrm{O}_{2}{ }^{-}\right)$, hydrogen peroxide $\left(\mathrm{H}_{2} \mathrm{O}_{2}\right)$, and hydroxyl radicals $(\mathrm{OH})$, which are byproducts of mitochondrial respiration. ROS is associated with oxidative stress and leads to different pathology by damaging lipids, proteins, or DNA. As seen in Fig. 4, the relative levels of superoxide and ROS were significantly higher in melanoma cells under suspension. Especially, superoxide level was 4.7-folded higher for suspended melanoma cells compared with that of adherent melanoma cells. On the other side, ROS level was 1.5-folded higher for suspended melanoma cells compared with that of adherent melanoma cells.

\section{Change in drug sensitivity of melanoma cells by cell detachment}

We demonstrated that the proliferation of adherent melanoma cells was more sensitive to the reduced glucose concentration (Fig. 1B) and were more glucose-dependent with higher glycolytic capacity. 2DG is the structural mimic of glucose that acts as a glycolysis inhibitor. As seen in Fig. 5A, increased 2DG 
concentrations resulted in decreased cell viability for adherent melanoma cells. However, increases in 2DG concentration had no significant effect on viability of suspended melanoma cells. On the other side, increase in maximal respiratory capacity and higher dependency on mitochondrial respiration in suspended melanoma cells would result in higher sensitivity toward blockade of electron transport chain. Rotenone is the inhibitor of complex I in electron transport chain. As seen in Fig. 5B, suspended melanoma cells were more sensitive to rotenone treatment. These results implied that detachment stress enabled metabolic switch from Warburg-like phenotype of higher glucose-dependency into anti-Warburglike phenotype of higher mitochondrial respiration-dependency. In summary, different drug sensitivities on metabolism were presented in adherent and suspended melanoma cells.

\section{Discussion}

The transformed cancer cells presented one specific feature of survival under detachment stress, which was beneficial for their metastatic spread. This feature of anchorage-independent survival is also called anoikis resistance. Previously, we had demonstrated the melanoma cells could survive under anchorage independency ${ }^{10}$. Detachment stress also modified the surface of malignant melanoma cells and affected their interactions with microenvironments. Reduced syndecan-1 expression impaired the laminin-binding ability and their ability of cell invasiveness ${ }^{10}$. This downregulation of syndecan- 1 was mediated by downregulation of aminopeptidase $\mathrm{N}$, which mediated by PKC $\delta$ activation and led to suppressed expression of integrin beta $4^{12}$. This generated different vascular phenotype of melanoma tumors. In addition, detachment stress also increased the expression of IL-8 and CXCR1 through activation of ERK and JNK signaling, that led to increased tumor growth ${ }^{11}$. Detachment stress also affected the chemosensitivity of melanoma cells through upregulation of syndecan-2-mediated ERK/PI3K activation ${ }^{13}$. These evidence all suggested the detachment stress would be one of the prime factors to transform the melanoma cells into different phenotype.

Some literatures suggested the reprogramming of metabolism enabled the cancer survival under the detachment stress ${ }^{18-20}$. The interplay between acidic microenvironment, ROS production, and HIF activation was closely associated with metabolic pathways in cancer cells ${ }^{20}$. It was suggested that low extracellular $\mathrm{pH}$ promoted anoikis resistance through an increased $\mathrm{N}$-cadherin expression with an enhanced migrative ability through the activation of epidermal growth factor receptor (EGFR) and Akt pathways. Our results showed the lactate secretion decreased as explained by the decreased expression of lactate transporter MCT4 (see Fig. 1C, 2A, and 2B). However, our unpublished data showed N-cadherin expression level increased upon cell suspension, upon which the lower level of extracellular acidification would achieve by decreased lactate secretion. In addition, the released lactate was shown to be angiogenic factor or metastatic factor through the action of IL-8/VEGF or CD44, respectively ${ }^{21}$. Our previous observation ${ }^{12}$ showed lower vascular phenotype in suspended cell-derived tumor, which could be explained by the decreased lactate secretion. 
It was reported that detachment stress causes mitochondria impairment and increased ROS production. The formation of cell clusters at suspension stage induced the HIF-1a activation and promoted glycolysis. Perturbation of these metabolic adaptations resulted in ROS accumulation and cell death ${ }^{22}$. It's also known the activation of transcription factor HIF-1 by lactate under hypoxia condition lead to tumor malignancy ${ }^{23}$.Our result (Fig. 4B) also showed increased HIF-1a expression under detachment stress, however, the portion of glycolytic routes was decreased (Fig. 3A and 3E). Production of superoxide might derive from either oxidation of NADPH by oxidase enzymes or from aerobic respiration in the mitochondria. Upon examination our previous microarray result (GSE42876), NOX4 expression was increased in 1.74-folded upon cell detachment.

According to our previous studies and current study, our conclusions suggested the detachment would actively promote the transformation of adherent melanoma cells in to different phenotypes at cell invasiveness, tumor malignancy, chemosensitivity, as well at altered metabolism of anti-Warburg feature. Although it's hard to said the suspended cells was dormant (less invasive, less proliferative, and less glycolytic) or less "transformed", we explained that detachment stress would enable the metastatic melanoma cells to survive under different environmental factors, such as immune system or chemotherapy.

\section{Methods}

\section{Cell culture and inhibitor treatments}

Human melanoma A375 cells were purchased from Bioresource Collection and Research Center (BCRC; Hsinchu, Taiwan) with authentication. Adherent culture was maintained using culture dish (Corning Incorporated Life Sciences, Tewksbury, Massachusetts, USA) and DMEM medium supplemented with 10\% (v/v) fetal bovine serum (Biological Industries Ltd., Cromwell, Connecticut, USA). Suspended melanoma cells were established and cultured by impaired attachment of adherent melanoma cells accordingly ${ }^{24,25}$. In general, $4 \times 10^{5}$ cells were cultured at sterile plastic dishes (Alpha-plus Inc., Taiwan) for 3 days before experiments. This generated the suspended melanoma cells.

Oligomycin (mitochondrial ATP synthase inhibitor), 2-deoxyglucose (2DG; glycolysis inhibitor), Trifluoromethoxy carbonylcyanide phenylhydrazone (FCCP; oxidative phosphorylation uncoupler), Rotenone and antimycin A (electron transport chain complex inhibitors) were all purchased from Sigma (Sigma-Aldrich China, Inc., Shanghai, PR China).

\section{Cell proliferation or cell viability assay}

For cell proliferation under different glucose concentrations, $4 \times 10^{5}$ cells were seeded at culture dish or sterile plastic dishes with indicated glucose concentration. Cells were incubated in humidified $\mathrm{CO}_{2}$ incubator for 3 days. The cells were collected and the total cell numbers were counted by trypan blue methods. 
For cell viability assay, it was assayed by alamar blue method (Thermo Fisher Scientific Inc., Pittsburgh, PA, USA). In general, $1 \times 10^{4}$ cells were seeded in each well of 96-well plate (Corning Incorporated Life Sciences, Tewksbury, Massachusetts, USA) pre-coated with or without polyHEMA. After overnight incubation, inhibitors were added and incubated for 1 days in culture incubators before the assays.

\section{Flow cytometry assay.}

For DNA content analysis by flow cytometry, the adherent and suspended cells were maintained at adherent or suspended condition respectively with indicated serum concentration for 3 days. The cells were then harvested by trypsinization, ethanol-fixed, and performed the propidium iodide staining in the standard procedure. The labeled cells were analyzed by Partec flow cytometer ML (Partec North America, Inc., NJ, USA).

\section{Lactate release assay and ROS detection assay}

Quantification of lactate level was done using Lactate Colorimetric Assay Kit (Biovision, Inc., Milpitas, California, USA) according to vendor's protocol. Adherent or suspended cells ( $2 \times 10^{5}$ cells) were incubated in the culture medium at $\mathrm{pH} 7.0$ or $\mathrm{pH} 8.5$ for 1 days without $\mathrm{pH}$ adjustment by $\mathrm{CO}_{2}$. The condition medium was collected and centrifuged for the following assay.

Detection of ROS levels in melanoma cells was done using ROS Detection Cell-Based Assay Kit (Cayman Chemical Co., Ann Arbor, Michigan, USA) according to vender's protocol. Dihydroethidium is the redoxsensitive fluorescent probe. Its oxidation into 2-hydroxyethidium by superoxide or other ROS causes the increased fluorescence intensity at $600 \mathrm{nM}$ or $576 \mathrm{~nm}$. Antimycin A treatment and $\mathrm{N}$-acetylcysteine treatment were positive and negative controls of ROS production, and used to calculate the relative percentage of ROS production.

\section{Polymerase chain reactions and statistical analysis}

The levels of mRNA in cultured cells were analyzed by qPCR. The cDNAs were synthesized by MMLV HP reverse transcriptase (Epicentre Inc., Madison, Wisconsin, USA) using freshly prepared RNA as PCR template. Quantitative real-time PCR was performed using Fast Quant Green Master Mix with ROX (Protech Technology Enterprise Co., Ltd., NanKung, Taipei, TAIWAN) in a StepOne Plus real-time PCR system (Thermo Fisher Scientific Inc., Pittsburgh, Pennsylvania, USA). The $2^{-\triangle \triangle C T}$ method was used to determine the relative gene expression using GAPDH as control. The $p$-value of $<0.05$ or $<0.01$ was statistically significant and was indicated in figures. The forward and reverse primers used were: MCT1 (SLC16A1), gtggctcagctccgtattgt and gagccgacctaaaagtggtg; MCT2 (SLC16A7), caacaccattccaagacagc and tggctgttatgtacgcagga; MCT4 (SLC16A3), cagttcgaggtgctcatgg and atgtagacgtgggtcgcat; GLUT1 (SLC2A1), ttgcaggcttctccaactggac and cagaaccaggagcacagtgaag; GAPDH, gagtcaacggatttggtcgt and gatctcgctcctggaagatg.

\section{Western blot and antibodies}


To harvest cell lysate for western blot analysis, cells were washed and disrupted by lysis buffer (10 mM Tris-HCl, 5 mM EDTA, pH 8.0, 1\% TritonX-100, and protease inhibitors) and kept on ice for $30 \mathrm{~min}$. The lysate was then centrifuged at maximum speed using a desktop centrifuge at $4^{\circ} \mathrm{C}$ for $15 \mathrm{~min}$. Protein concentrations were quantified by protein assay kit (Bio-Rad Laboratories Inc., Hercules, California, USA).

Western blot was performed according to standard protocol. Briefly, the protein mixture was subjected to SDS-PAGE and transferred onto a PVDF membrane followed by blocking with $5 \%(\mathrm{w} / \mathrm{v})$ skim-milk. The membrane was then incubated in primary antibodies (1:1000 in 5\% skim-milk in TBST) overnight at $4{ }^{\circ} \mathrm{C}$, and HRP-conjugated secondary antibody $(1: 20000)$ for $1 \mathrm{hr}$ at room temperature followed by enhanced chemiluminescent (Millipore Co., Massachusetts, USA) detection. The primary antibody against MCT1 (SLC16A1), MCT2 (SLC16A7), MCT4 (SLC16A3), GLUT1 (SLC2A1) and $\beta$-actin were all purchased from GeneTex Inc., Hsinchu, Taiwan.

\section{Metabolism stress tests using Seahorse XF24 analyzer}

The mitochondrial oxygen consumption rate (OCR) and the extracellular acidification rate (ECAR) were measured using a Seahorse Bioscience XF24 extracellular flux analyzer (Agilent Technologies, Inc., Santa Clara, California, USA).

To prepare adherent melanoma cells, $5 \times 10^{4}$ cells were seeded at Seahorse cell culture microplate for overnight and reached $100 \%$ confluency on the next day. To prepare suspended melanoma cells, $5 \times 10^{4}$ suspended cells were seeded on the experiment day at microplate precoated with poly-L-lysine. The sensor cartridge with the utility plate was assembled and incubated in the non- $\mathrm{CO}_{2}$ incubator at $37^{\circ} \mathrm{C}$ overnight before the assays.

For mitochondria stress test to measure OCR, culture medium was replaced and incubated with glucosefree/ serum-free/bicarbonate-free medium (D5030; Sigma-Aldrich China, Inc., Shanghai, PR China) for 1 $\mathrm{hr}$ before the assays. Mitochondria stress profiles were monitored after the successive injection of oligomycin $(2 \mu \mathrm{M})$, FCCP (carbonyl cyanide p-trifluoromethoxyphenylhydrazone, $1 \mu \mathrm{M}$ ), and rotenone/antimycin A ( $1 \mu \mathrm{M}$ each) to the indicated wells. Glycolysis stress profiles were monitored after the successive injection of glucose $(10 \mathrm{mM})$, oligomycin $(2 \mu \mathrm{M})$, and 2-deoxyglucose (2DG, $50 \mathrm{mM})$. OCR and ECAR results were analyzed using the Seahorse XF-24 software. Every point represents an average of three different wells. The cell numbers in each wells were counted after the experiments, that were used to normalize the ECAR and OCR.

\section{Declarations}

\section{ACKNOWLEDGMENTS}

This work was supported by grant 106-SKH-FJU-07 and 108-SKH-FJU-05 from Shin Kong Wu Ho-Su Memorial Hospital. 


\section{AUTHOR CONTRIBUTION}

WCU acquired information, edited manuscript, and finalized manuscript; TTT and CPW designed and performed experiments; SCL designed experiments, supervised this study, wrote manuscript, and finalized manuscript; All authors read and approved the final manuscript.

\section{COMPETING INTERESTS}

The author(s) declare no competing interests.

\section{References}

1. Kim, Y. N., Koo, K. H., Sung, J. Y., Yun, U. J. \& Kim, H. Anoikis resistance: an essential prerequisite for tumor metastasis. Int J Cell Biol 2012, 306879, doi:10.1155/2012/306879 (2012).

2. Okayama, H. Cell cycle control by anchorage signaling.Cell Signa/24,1599-1609, doi:S08986568(12)00120-9 [pii]

3. 1016/j.cellsig.2012.04.005 (2012).

4. Zhong, X. \& Rescorla, F. J. Cell surface adhesion molecules and adhesion-initiated signaling: understanding of anoikis resistance mechanisms and therapeutic opportunities.Cell Signa/24,393401, doi:S0898-6568(11)00333-0 [pii]

5. 1016/j.cellsig.2011.10.005 (2012).

6. Simpson, C. D., Anyiwe, K. \& Schimmer, A. D. Anoikis resistance and tumor metastasis. Cancer letters, 272, 177-185 https://doi.org/10.1016/j.canlet.2008.05.029 (2008).

7. Vander Heiden, M. G., Cantley, L. C. \& Thompson, C. B. Understanding the Warburg effect: the metabolic requirements of cell proliferation.Science324,1029-1033, doi:324/5930/1029 [pii]

8. 1126/science.1160809 (2009).

9. Levine, A. J. \& Puzio-Kuter, A. M. The control of the metabolic switch in cancers by oncogenes and tumor suppressor genes.Science330,1340-1344, doi:330/6009/1340 [pii]

10. 1126/science.1193494 (2010).

11. Cairns, R. A., Harris, I. S. \& Mak, T. W. Regulation of cancer cell metabolism.Nat Rev Cancer11,85-95, doi:nrc2981 [pii]

12. $1038 /$ nrc2981 (2011).

13. Dang, C. V. Links between metabolism and cancer.Genes Dev26,877-890, doi:26/9/877 [pii]

14. 1101/gad.189365.112 (2012).

15. Soga, T. Cancer metabolism: key players in metabolic reprogramming. Cancer Sci, 104, 275-281 https://doi.org/10.1111/cas.12085 (2013).

16. Wang, C. et al. Loss of cell invasiveness through PKC-mediated syndecan-1 downregulation in melanoma cells under anchorage independency. Experimental dermatology, 23, 843-849 https://doi.org/10.1111/exd.12550 (2014). 
17. Uen, W. C. et al. Anchorage independency promoted tumor malignancy of melanoma cells under reattachment through elevated interleukin-8 and CXC chemokine receptor 1 expression. Melanoma research, 25, 35-46 https://doi.org/10.1097/CMR.0000000000000134 (2015).

18. Cheng, Y. C. et al. Anchorage independence altered vasculogenic phenotype of melanoma cells through downregulation in aminopeptidase N /syndecan-1/integrin beta4 axis. Aging (Albany NY), 12, 16803-16819 https://doi.org/10.18632/aging.103425 (2020).

19. Tseng, T., Uen, W., Tseng, J. \& Lee, S. Enhanced chemosensitization of anoikis-resistant melanoma cells through syndecan-2 upregulation upon anchorage independency. Oncotarget, 8, 61528-61537 https://doi.org/10.18632/oncotarget.18616 (2017).

20. Ippolito, J. E. et al. Extracellular pH Modulates Neuroendocrine Prostate Cancer Cell Metabolism and Susceptibility to the Mitochondrial Inhibitor Niclosamide. PloS one, 11, e0159675 https://doi.org/10.1371/journal.pone.0159675 (2016).

21. Doherty, J. R. \& Cleveland, J. L. Targeting lactate metabolism for cancer therapeutics. J Clin Invest, 123, 3685-3692 https://doi.org/10.1172/JCl69741 (2013).

22. van der Windt, G. J. et al. Mitochondrial respiratory capacity is a critical regulator of CD $8+T$ cell memory development., 36, 68-78 https://doi.org/10.1016/j.immuni.2011.12.007 (2012).

23. Zhang, J. et al. Measuring energy metabolism in cultured cells, including human pluripotent stem cells and differentiated cells. Nat Protoc, 7, 1068-1085 https://doi.org/10.1038/nprot.2012.048 (2012).

24. Hawk, M. A. \& Schafer, Z. T. Mechanisms of redox metabolism and cancer cell survival during extracellular matrix detachment. The Journal of biological chemistry, 293, 7531-7537 https://doi.org/10.1074/jbc.TM117.000260 (2018).

25. Mason, J. A., Hagel, K. R., Hawk, M. A. \& Schafer, Z. T. Metabolism during ECM Detachment: Achilles Heel of Cancer Cells? Trends Cancer, 3, 475-481 https://doi.org/10.1016/j.trecan.2017.04.009 (2017).

26. Adeshakin, F. O. et al. Mechanisms for Modulating Anoikis Resistance in Cancer and the Relevance of Metabolic Reprogramming. Front Oncol, 11, 626577 https://doi.org/10.3389/fonc.2021.626577 (2021).

27. Dhup, S., Dadhich, R. K., Porporato, P. E. \& Sonveaux, P. Multiple biological activities of lactic acid in cancer: influences on tumor growth, angiogenesis and metastasis. Curr Pharm Des, 18, 1319-1330 doi:CPD-EPUB-20120223-008 [pii] (2012).

28. Labuschagne, C. F., Cheung, E. C., Blagih, J., Domart, M. C. \& Vousden, K. H. Cell Clustering Promotes a Metabolic Switch that Supports Metastatic Colonization. Cell Metab 30, 720-734 e725, doi:10.1016/j.cmet.2019.07.014 (2019).

29. Sonveaux, P. et al. Targeting the lactate transporter MCT1 in endothelial cells inhibits lactate-induced HIF-1 activation and tumor angiogenesis.PLoS One7,e33418, doi:10.1371/journal.pone.0033418

30. PONE-D-11-24085 [pii] (2012). 
31. Wang, J. Y. et al. Impaired homologous recombination DNA repair and enhanced sensitivity to DNA damage in prostate cancer cells exposed to anchorage-independence.Oncogene24,3748-3758, doi:1208537 [pii]

32. 1038/sj.onc.1208537 (2005).

33. Al-Ayoubi, A., Tarcsafalvi, A., Zheng, H., Sakati, W. \& Eblen, S. T. ERK activation and nuclear signaling induced by the loss of cell/matrix adhesion stimulates anchorage-independent growth of ovarian cancer cells. J Cell Biochem, 105, 875-884 https://doi.org/10.1002/jcb.21889 (2008).

\section{Figures}


Figure 1

A
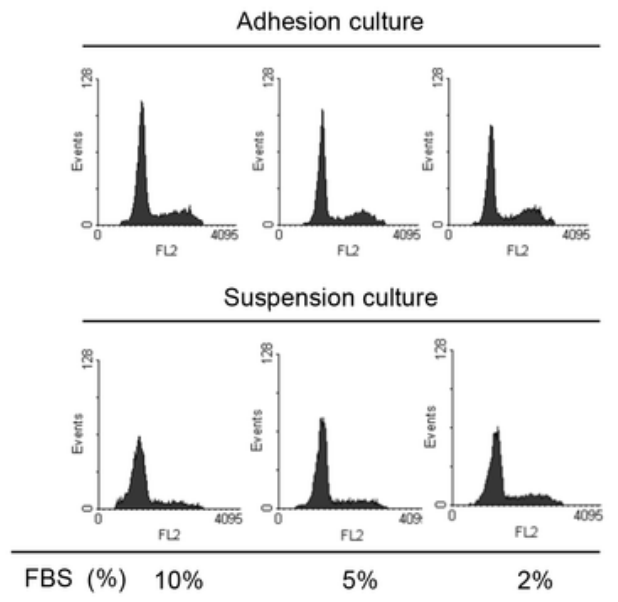

B

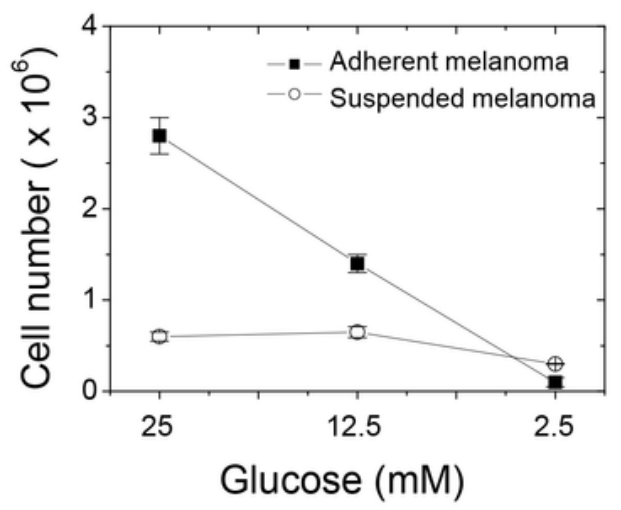

C

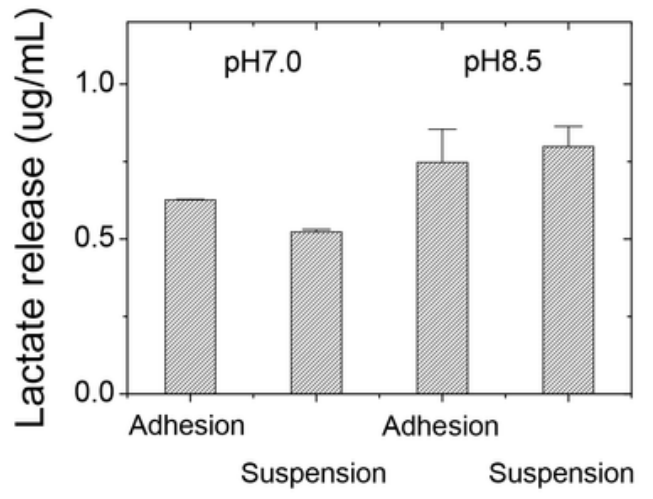

\section{Figure 1}

Detachment stress contributed to the characteristics of slow proliferation, resistance to glucose depletion, and decrease lactate secretion. A) DNA content analysis showed that suspension culture reduced G2 contents as assayed by flow cytometry. B) Proliferation of adherent melanoma cells showed decreased number by decreased glucose concentrations, while suspended melanoma cells were relatively unaffected. C) Reduced lactate secretion from suspended melanoma cells was seen under neutral pH 
condition (pH7.0). Under pH8.5, the levels of lactate secretions were the same for adherent and suspended melanoma cells.

Figure 2

A

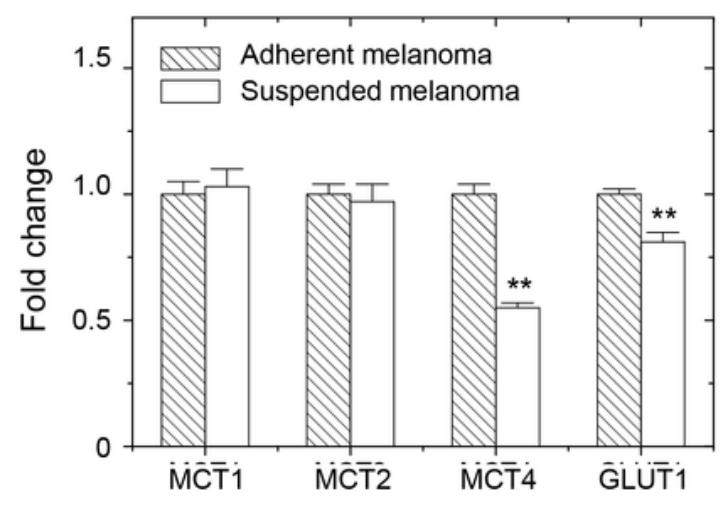

B

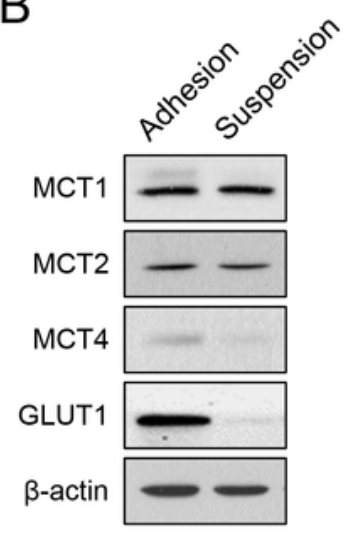

Figure 2

Decreased MCT4 and GLUT1 expression in suspended melanoma cells as examined by A) qPCR and B) western blot. 
Figure 3
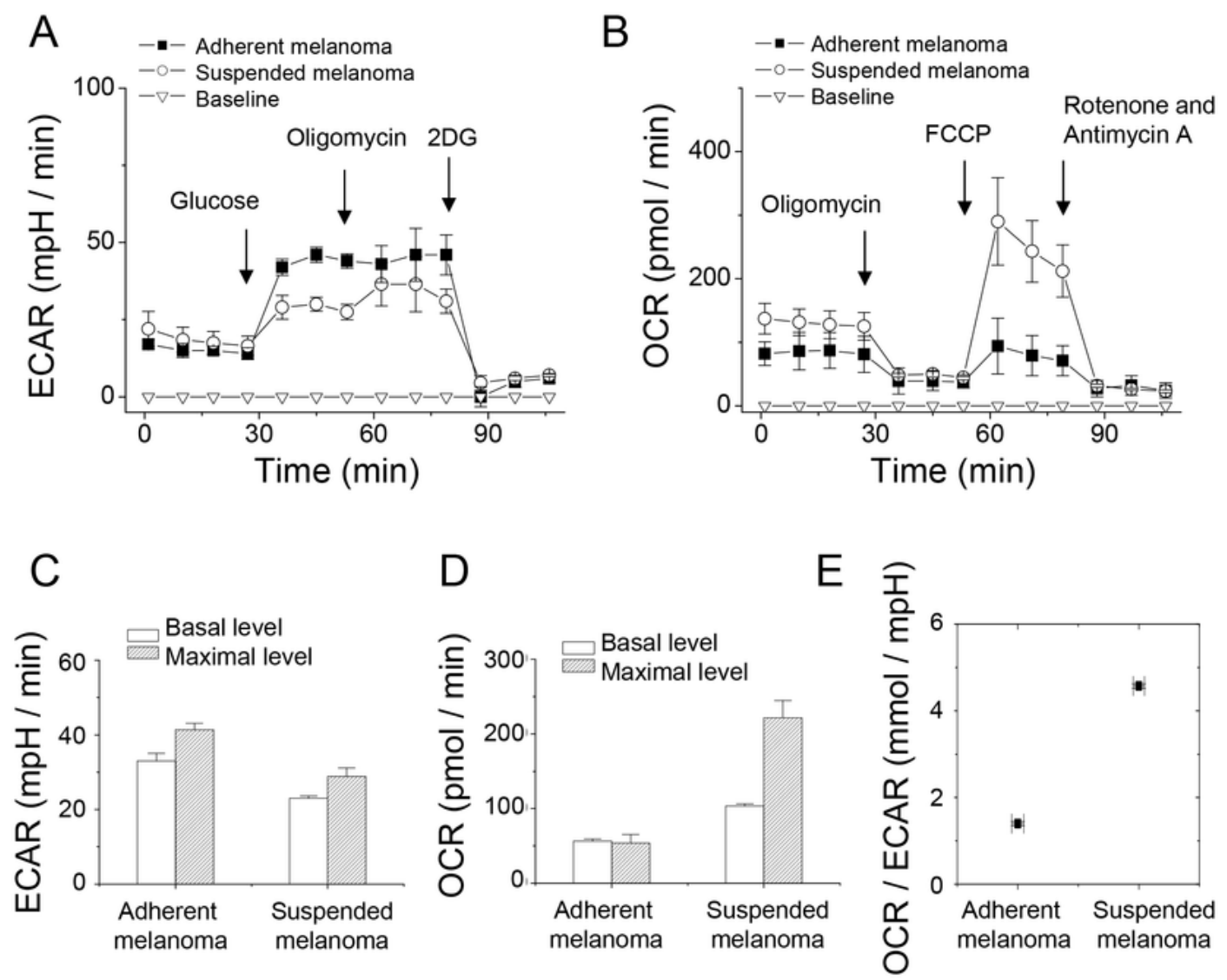

\section{Figure 3}

Detachment stress decreased glycolytic capacity but increased respiratory capacity of melanoma cells investigated by Seahorse XF24 analyzer. A) ECAR analysis showed higher rate of glycolysis in adherent melanoma cells. B) OCR analysis showed higher oxygen consumption in suspended melanoma cells. C) Decreased glycolytic capacity was observed in suspended melanoma cells, but 80\% glycolytic capacities were used in both adherent and suspended melanoma cells. D) Increased respiratory capacity 
was seen in suspended melanoma cells. Adherent melanoma cells used full capacity in oxygen consumption, while suspended melanoma cells used only $46.7 \%$ of capacity. E) Metabolism in suspended melanoma cells was highly dependent on mitochondrial respiration.

Figure 4

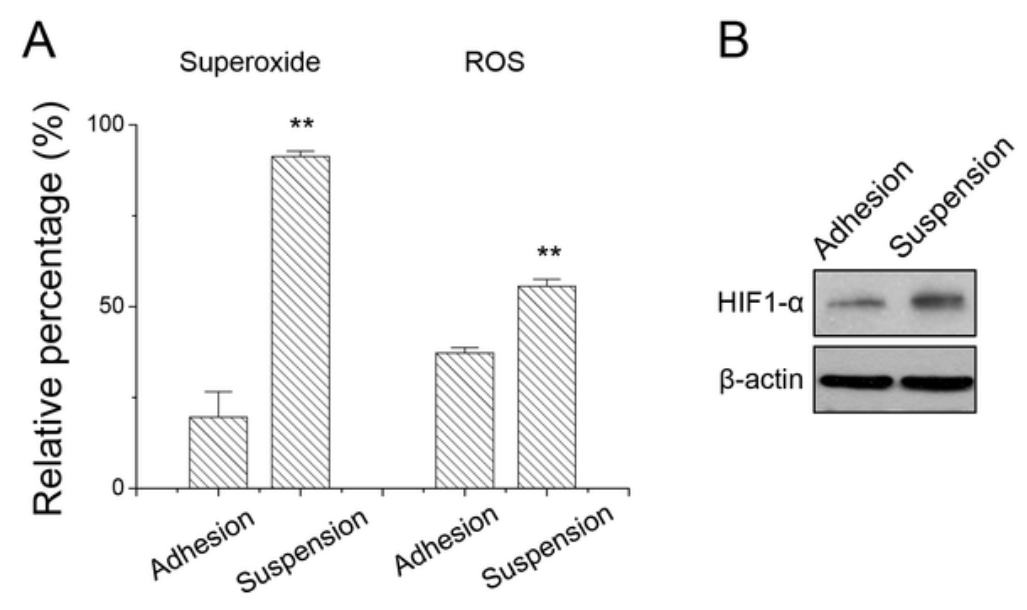

Figure 4

Detachment stress increased the superoxide and ROS production. 
Figure 5

A

B
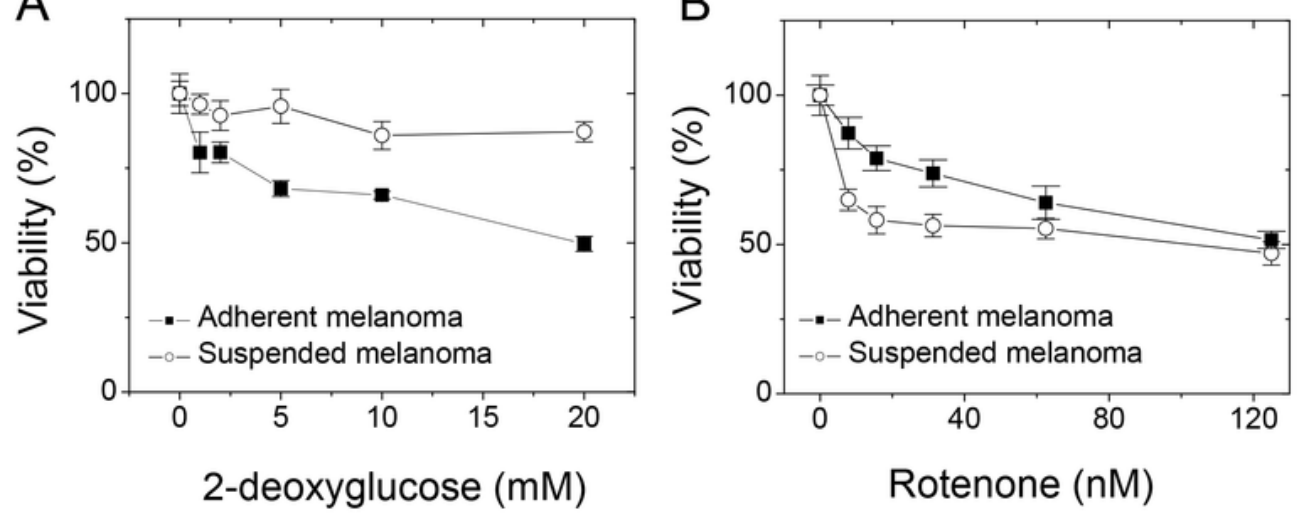

\section{Figure 5}

Detachment stress altered the drug sensitivities toward the blockades of glycolysis and mitochondrial respiration. Suspended melanoma cells were less sensitive to $A$ ) treatment of $2 D G$, but more sensitive to B) treatment of rotenone. 